\title{
DIGITAL SKETCH MODELLING: PROPOSING A HYBRID VISUALISATION TOOL COMBINING AFFORDANCES OF SKETCHING AND CAD
}

\author{
Ranscombe, Charlie (1); Zhang, Wenwen (1); Rodda, Jacob (1); Mathias, David (2) \\ 1: Swinburne University of Technology; 2: Bristol University
}

\begin{abstract}
Visualisation of ideas and emergent designs is an essential ingredient in design practice. Sketching and CAD represent two widely used visualisation tools, each with complementary affordances that dictate their typical use during the design process. Sketching has affordances of fast and fluent visualisation whereas CAD affords easy modification of detailed designs. This paper proposes a hybrid tool, Digital Sketch Modelling, investigating the extent to which it can deliver complementary affordances of both sketching to CAD. Analysis of diary entries made by 62 postgraduate designers using sketching, digital sketch modelling and CAD within a design project forms the basis of the study. Results illustrate how digital sketching over crude $3 \mathrm{~d}$ digital models, combined with benefits of digital image editing software enhance affordance for easy visualisation of ideas. Concurrently, the level of software used in Digital Sketch modelling led to fewer concerns over the level of difficulty to modify designs, enhancing the affordance for easy modification. As such we conclude Digital Sketch Modelling does combine affordances indicating its potential benefit in use between sketching and CAD.
\end{abstract}

Keywords: Sketching, Computer Aided Design (CAD), Visualisation, Communication, Affordances

\section{Contact:}

Ranscombe, Charlie

Swinburne University of Technology

Architectural and Industrial Design

Australia

cranscombe@swin.edu.au

Cite this article: Ranscombe, C., Zhang, W., Rodda, J., Mathias, D. (2019) 'Digital Sketch Modelling: Proposing a Hybrid Visualisation Tool Combining Affordances of Sketching and CAD', in Proceedings of the 22nd International Conference on Engineering Design (ICED19), Delft, The Netherlands, 5-8 August 2019. DOI:10.1017/dsi.2019.34 


\section{INTRODUCTION}

Visualisation of emergent designs is an essential part of the product development process (Cross 1982; Lawson 2002). Currently there is a vast array of digital design tools available to industrial or product designers and engineers, to support the visualisation and subsequent reflection and exploration of design solutions (Aldoy and Evans 2011). For example, visualisations can range from crude sketches on the back of an envelope to fully detailed technical drawings, 3D digital models or photorealistic renderings of designs.

Use of different tools can influence the nature of the resulting design due to the relative affordances of tools at different points in the design process (Tsai and Yang 2017). It follows that affordances of different tools dictate their use as more suited to different phases of the design process. The two tools at the focus of this study Sketching and CAD are prime examples of tools with complementary affordances. Sketching has strengths in fast and fluent visualisation (Goldschmidt 1991) however is weaker in modification of more resolved designs, and resulting visualisation are usually less detailed. CAD has strengths in accurate visualisation and easy modification of designs (J. A. Self 2013). However, CAD can be slower and potentially difficult to use earlier in design process when designs are less comprehensive, due to many CAD packages requiring precise measurements to create visualisations (Lawson 2002). These relative strengths are illustrated in Figure 1 with respect to the phases in the design process where they are typically used.
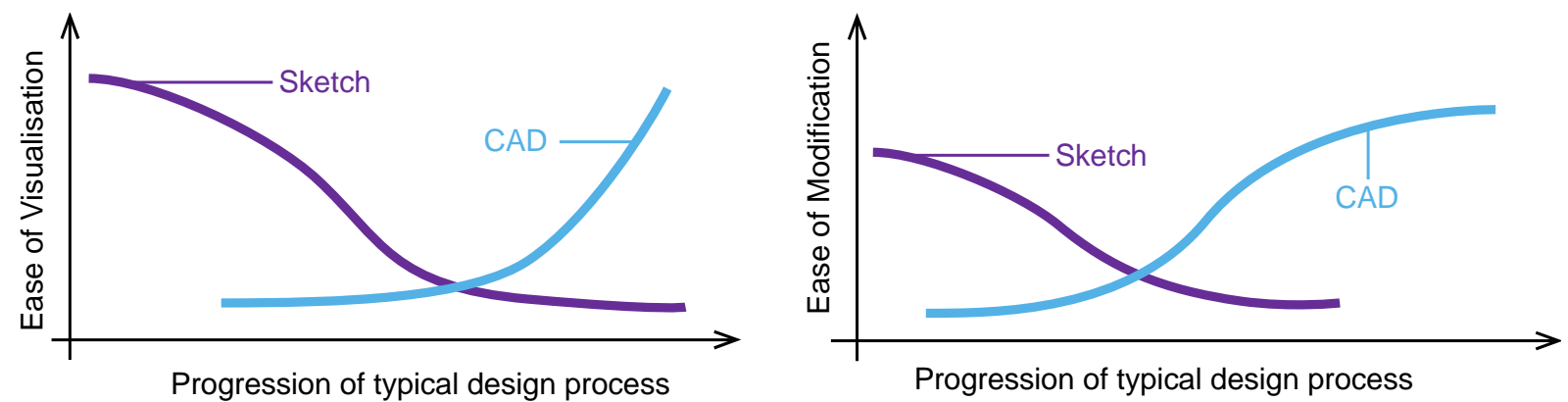

Figure 1. Illustrating relative complementary affordances of Sketching and CAD during the progression of a typical product design process

Due to the complementary nature of the affordances for easy visualisation and easy modification, there is a point during the design process where designers transition from primarily using sketching to using CAD. At this point of transition neither the use of sketching nor CAD is optimal, creating difficult transitions between the primary use of one tool to another. Note, we emphasise primary use understanding that there is often an overlap where designers use both tools.

To ease this transition, we believe there is scope to create a hybrid visualisation tool that couples the complementary affordances of sketching and CAD. This would be used as an interim tool between sketching and CAD with the goal of making the transitions between sketching and CAD smoother and less challenging resulting in a more seamless design process. Thus, the aim of the research reported here is investigating the extent to which Digital Sketch Modelling (DSM) as a hybrid tool can deliver the complementary affordances of sketching and CAD.

The remainder of this paper is structured as follows. Section 2 gives a summary of characteristics of visualisation tools as well as overviews of existing research proposing hybrid visualisation tools and Digital Sketch Modelling (DSM, the tool studied in this paper). Section 3 explains the research method used, and section 4 presents key results and discussion. Limitations of the study are given, and further work described in section 5 and conclusions drawn in section 6 .

\section{BACKGROUND}

\subsection{Characteristics of typical design tools}

As stated above, there are substantial differences across existing design tools. In order to draw comparisons between new hybrid design tools and existing design tools, we must first define a framework of design tool characteristics (DTCs) that are generic across all tools. For the purpose of 
this study we take the framework proposed by Zhang et al. (2019). This framework builds on highly cited frameworks by (Purcell and Gero 1998) and (J. Self et al., 2009) offering a comprehensive list of key characteristics of design tools that influence their affordances and utility within the design process. Table 1 gives a list of the DTCs.

Table 1 Summary of Design Tool Characteristics outlined in Zhang et al., 2019 used as the basis for comparisons in this study

\begin{tabular}{|l|l|l|}
\hline Ambiguity & Mobility & Learning Cost \\
Internal Communication & Immediacy & Using Cost \\
External Communication & Flexibility & Emotional Commitment to \\
Problem Re-framing & Level of Details & Ideas Expertise \\
Lateral Transformation & Level of Aesthetics & User Accessibility \\
Vertical Transformation & Amount of Renderings & User Loyalty \\
Level of Commitment & Holistic View of Objects & User Share \\
Fidelity & Compatibility & \\
\hline
\end{tabular}

\subsection{Existing hybrid sketch and CAD tools}

Since the emergence of CAD software, practices and terminology from traditional design tools have been brought into software. Beyond technical developments there has been sustained academic research intending to blend CAD with sketching since the early 2000's. Bae et al. (2003) studied designers' sketches to develop methods to convert sketches into 3D geometry. Levent Burak Kara et al. (2006) and L B Kara and Shimada (2008), acknowledging the advantages of sketching and pen based input, go further by creating software that interprets pen based strokes made on a digital interface. Similarly, Company et al. (2009) also explore digital interpretation of paper sketches in 3D, but notably differentiate between thinking, prescriptive and talking types of sketches. More recently this interpretation has been advanced by combining with generative design to produce variant designs derived from sketches (Orbay and Kara 2012). We can summaries the above mentioned research as creation of design software that attempts to integrate the affordances of sketching with the intention of reducing design time and thus improving efficiency.

Developers of commercial CAD software have to some extent realised the above research creating various innovative visualisation software platforms. These are both screen based (Zammit and Munoz 2014) and more recently incorporate virtual and augmented reality (Arora et al., 2018), some of which form the basis of academic research in design. A relevant example is Alcaide-Marzal et al. (2013) that explores the use of digital sculpting as an alternative to more rigid parametric CAD packages. Digital sculpting aims to emulate the freeform input of physically moulding clay, representing a hybrid design tool that is skewed towards CAD modelling. Said study finds that despite the freedom afforded by MudBox, the flexibility is still far from paper and sketch. Research by Evans et al. (2015) investigates more broadly the suitability of an entirely digital industrial design workflow. In this study Evans et al. (2015) coin the term "Digital Sketch model", describing the creation of early 3D forms which are edited in sketch-like environment. This concept of digital sketch modelling is taken further by Ranscombe and Bissett-Johnson (2017) embodying it in a workflow that blends sketching with CAD via a digital sketching medium (full description of the workflow is given below). The digital sketch model stands out as it embodies a hybrid tool that is a true blend of sketching and CAD. In other words, it mixes 3D geometry with a sketch-based interface as opposed to interpreting sketches made by hand or attempting to emulate sketching through 3D modelling software features.

The specifics of the Digital Sketch Modelling (DSM) tool used in the study reported are derived from Ranscombe and Bissett-Johnson (2017), the reader is directed to the original source for a detailed description of the tool. In summary DSM is a hybrid of sketching and CAD that, brings basic CAD (rudimentary 3D model) into a digital sketching environment affording the user the ability to sketch ideas over the top of the basic CAD model termed "proxy model". Thus, DSM presents a hybrid where the user is sketching but makes use of simplified CAD geometry alongside digital functionality like being able to undo, resize. 


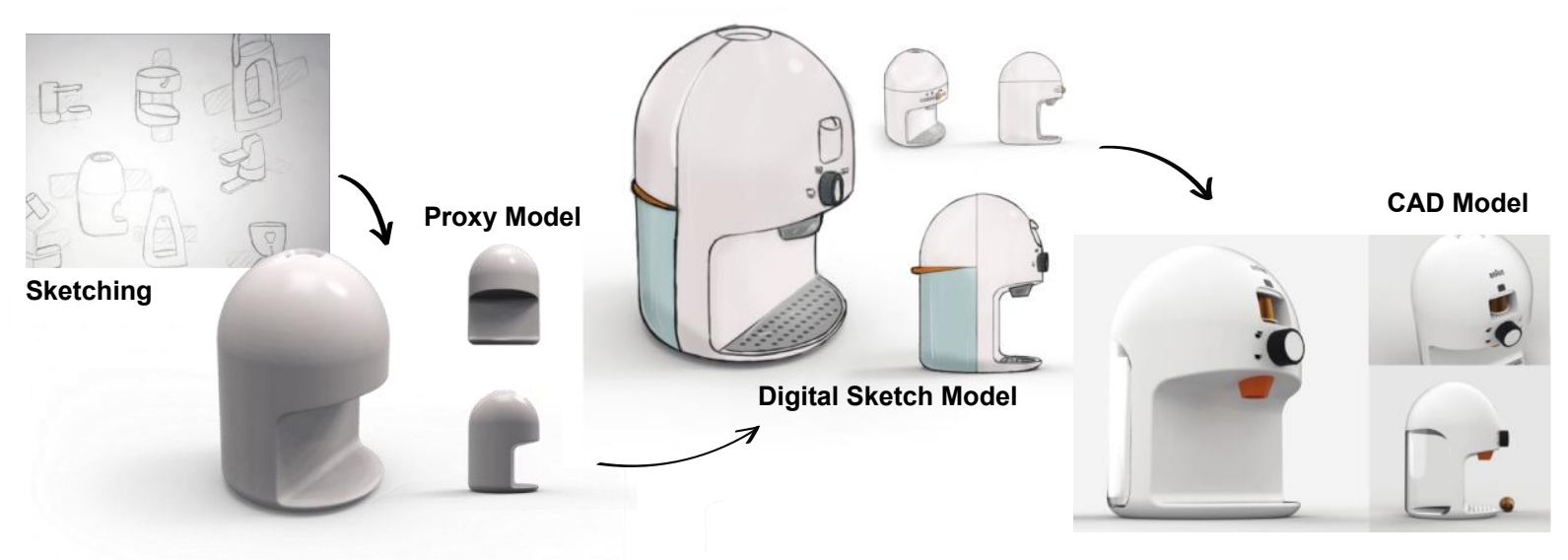

Figure 2. Examples of work resulting from the study presented illustrating the key steps that make up the Digital Sketch Modelling tool

The primary rationale for adopting DSM is that it is pitched at a level of detail between rough sketch and CAD. This makes it suited for use during the transition between Sketching and CAD. Furthermore, the tool can be used adopting different software, all that is required is a 3D modelling software and $2 \mathrm{~d}$ image editing/drawing software. For the purpose of this study Rhinoceros 3D was selected as the CAD software and Adobe Photoshop used as the image editing software. This is important logistically to be able to study the workflow, but also important for future research as it offers the possibility to discover develop knowledge that is generalisable beyond specific or bespoke software developed by researchers. Digitising tablets were also provided to offer stylus/pen-based sketch input within Photoshop.

\section{METHOD}

To investigate the extent to which DSM as a hybrid visualisation tool can deliver the complementary affordances of sketching and CAD, we created a study to trial DSM within a student design process alongside traditional sketching and CAD. The sample of designers was comprised of 62 Masters of Design students. Masters level was selected to ensure all students had a degree of familiarity with design theory, process, and the tools used and hence form a level of design competence comparable to graduate/entry level designers. The study was undertaken as part of a digital ideation elective unit within the Master's degree.

\subsection{Design task}

The task for the study was focused around a typical industrial or product design activity representative of early to middle stages of the product design process. Participants were given a brief whereby they assumed the role of designers for an appliance company tasked with redesigning a pod coffee machine to use existing internal components but aligning the product shell with styling of another company (Braun or Cadillac). The end result of the task was design modelled in CAD to be submitted alongside a portfolio showing progression of the design in form of the different visualisations used to achieve the final design.

\subsection{Design process}

A 7-week design process followed by participants was scaffolded to simulate a typical product design process. Participants were directed to spend the first week in an ideation phase using Sketching to explore a wide range of ideas (approx. 1 week). Following this, participants spent approx. 3 weeks using DSM to further conceptualise 2-3 of their initial ideas. Finally, participants spent the remaining 3 weeks using CAD to develop and refine a final design using CAD.

\subsection{Data collection}

The source of data used to investigate DSM was a diary recorded by each participant as part of their design project folio. The diary method was adopted based on its use in similar studies (Badke-Schaub 
and Frankenberger 1999; Evans et al., 2015; Pedgley 2007), citing advantages in capturing designers' multiple reflections on their own practice over the duration of a design task. For the study reported in this paper we focus on responses during the course of the project to the following two questions:

"How difficult did you find using the tool(s) to convert ideas in your head into visualisations?"

"How easy did you find the tools used it to modify or change your designs as your ideation progressed?"

Question one is posed to direct comments on the affordance for easy visualisation commonly held for sketching. Question two to direct comments on the affordance for easy modification commonly seen as having advantage of CAD. Participants were instructed to write a minimum of 3 diary entries ensuring at least one entry covering each tool and phase of the design process.

\subsection{Data analysis}

Diary entries responding to the above questions were coded as follows. First entries were coded to ascertain the tool discussed. If this was not clear from diary entries, design work also included in folios was reviewed to confirm which tool was being referred to. Next entries were coded in terms of the DTCs being cited with respect to ease of visualisation and ease of modification. Finally, the reference to each DTC was coded as to whether the DTC was cited in a positive or negative attitude. Positive attitudes are defined as attitudes indicative of facilitating easy visualisation/modification of ideas. Negative attitudes are indicative of barriers to easy visualisation/modification of ideas. Coding was conducted separately by two researchers to reduce bias in interpreting diary entries. Table 2 gives an example of diary entry coding.

Table 2. Example coding scheme used to analyse diary entries

\begin{tabular}{|l|l|l|l|l|}
\hline Question & Diary Entry & Tool & DTCs & Attitude \\
\hline Ease of & $\begin{array}{l}\text { "Difficult because I don't have a sketching } \\
\text { Vackground transforming proxy models into } \\
\text { concepts and knowing where to place lines is } \\
\text { hard. Rhino is not too bad once a few } \\
\text { techniques are learned." }\end{array}$ & $\begin{array}{l}\text { Fidelity } \\
\text { Expertise }\end{array}$ & $\begin{array}{l}\text { Negative } \\
\text { Positive }\end{array}$ \\
\hline
\end{tabular}

\section{RESULTS AND DISCUSSION}

Results are presented first with an overview of positive and negative statements made in response to easy visualisation and modification when using each of the design tools (4.1). The majority of this section is then dedicated to more detailed review of the most frequently cited characteristics referenced with respect to ease of visualisation (4.2) and ease of modification (4.3).

\subsection{Overview: easier or harder to visualise and modify ideas}

To establish whether DSM delivers positive experience in affordances of easy visualisation and easy modification, the number of positive and negative attitudes in diary entries when using each tool are compared in Figure 3. In terms of easy visualisation of ideas, Figure 3 shows the use of DSM described $60 \%$ positive attitudes versus $27 \%$ negative (the remainder being nonpartisan). Compared with 53:43 for Sketch and 45:41 for CAD, this shows that DSM is presents a comparatively easier means to visualise ideas.

In terms of easy modifying of ideas, Figure 3 shows diary entries discussing DSM were positive in approximately the same proportion as CAD (DSM 57:32 and CAD 53:36 positive and negative), but that entries discussing sketching showed far higher proportion of positive attitudes, $76 \%$ positive versus $22 \%$ negative. Thus, from this data set DSM presents at the same level of ease of modification compared with $\mathrm{CAD}$, thus seen as less easy to use in terms of modification compared with sketching. 


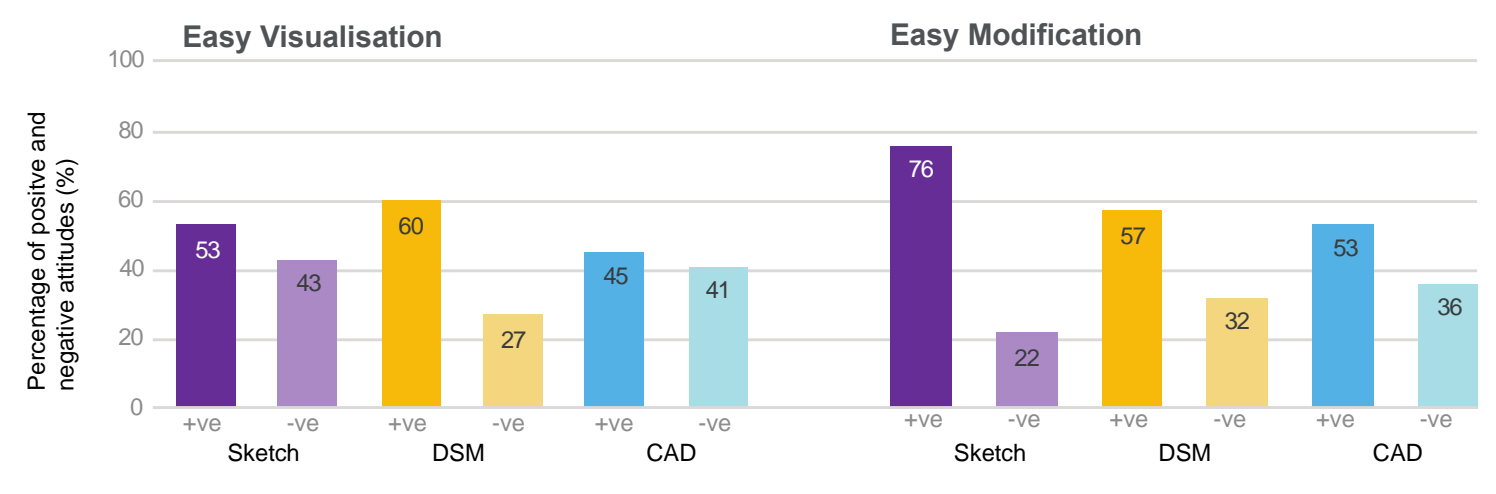

Figure 3. Percentage of positive versus negative attitudes with respect to easy visualisation and easy modification compared across sketching, DSM, and CAD

\subsection{Characteristics (DTCs) cited with respect to ease of visualising ideas}

Figure 4 presents data on the most frequently cited characteristics in diary entries referring to ease of visualising. Charts show for each tool the total citations of each characteristic alongside the proportion of positive vs negative attitudes.

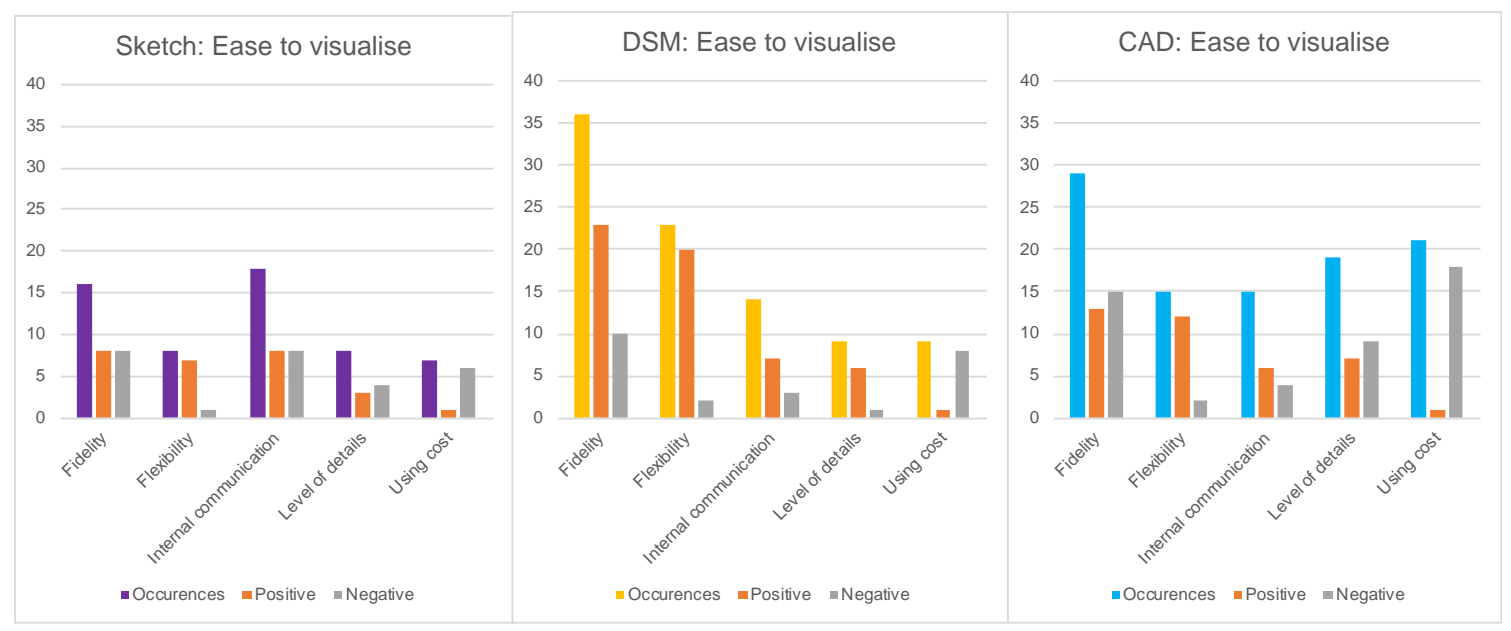

Figure 4. Comparison of most frequently cited DTCs along with respective proportion of positive versus negative attitudes describing each DTC on the topic of easy visualisation

Fidelity stands out as having a positive influence on easy visualisation as it was cited most frequently in terms of ease of visualisation for students using DSM. Comparing responses citing fidelity it can be seen that of 36 citations of Fidelity, 23 are positive, 10 negative and 3 neutral when using DSM. Comparatively there are 16 citations for Sketching and 29 citations for CAD with 8:8 and 13:15 positive: negative respectively.

Comments for DSM on the characteristic of Fidelity discuss the influence of the proxy model, highlighting the level of fidelity afforded as helpful to the process of visualising ideas. For example: Participant 14 comments: "Sketching over a proxy model on the screen is a lot easier than without a model since there is a guideline". Similarly, Participant 18 comments: "It's not so hard to do sketchover on the proxy models since I already had a structure that my sketches can be based on." Where those using DSM had negative comments relating to Fidelity, attitudes related to familiarity with software. For example, Participant 32 had a mixed experience, commenting, "Both easy and difficult. It's still a great challenge to visualize ideas especially when I have to make the ideas more specific. But with Photoshop assisted with Rhino I'm slowly but surely doing progress."

Participant 57's comment exemplifies a typical negative attitude with respect to fidelity and visualising ideas. "It was quite difficult at this stage to convert the very artistic fancy ideas in my head into 3D". This may be explained by other comments highlighting familiarity with software as the driving the experience. For example, Participant 60 had an overall positive experience visualising their design using CAD citing progressing familiarity with the software, "I'm finding different and easier ways to things like using curves and extruding and revolving instead of only working with solids. This 
makes it easier to recreate the idea I have in my head. Overall, I feel like the pace and ease of my workflow is essential and ultimately decides how smooth the conversion from idea to visualisation is." Flexibility is cited second most frequently in all 3 tools. Here citations of the Flexibility characteristic are positive and negative in approximately equal proportions. Hence while data shows the Flexibility of the tool is priority for easy visualisation, comparison does not indicate that one tool is significantly more positively flexible. Reviewing comments in more detail shows some patterns in the way that the tools lead/afford positive attitudes to Flexibility. For DSM attitudes focused on Flexibility afforded by the combination of software/tools. For example, Participant 39 comments, "Mixing Photoshop with the proxy models give me more [freedom]..." where negative attitudes were recorded they tended to relate to the downside of multiple software, namely complexity of iterating between two software, for example, "It took long for me to edit the sketchover with Photoshop since I wanted to adjust the proxy models."

However, when discussing CAD, we can see further the downsides of software for ease of visualisation. For example, Participant 56 comments, "Complicated to figure out how to use the proper tools when modelling". CAD diary entries discussing software citing the characteristic Using cost are also relevant here. They show a primarily negative attitude, with many also highlighting software as the source of substantial time spent in creating models. For example, the comment by Participant 62 "I found it difficult because even if I learned how to use some tools in the software, I don't have total command of it and sometimes it limited the design process."

\subsection{Characteristics (DTCs) cited with respect to ease of modifying ideas}

Figure 5 presents data on the most frequently cited characteristics with respect to ease of modification outlining the proportion of positive and negative citations. Flexibility is most frequently cited in terms of easy modification of designs. Of those occurrences, it is heavily skewed to the positive in sketching 19 vs 3. DSM is also skewed positively 27:10 and then CAD 20:12. Further review shows that many diary entries on DSM citing characteristics of flexibility describe software as an enabler (positive) of easy modification. For example, Participant 60 comments when discussing both sketching and DSM, "I think hand-sketching can be a good control of the lines, but the Photoshop (PS) tool can save me a lot of time of redrawing. For example, I drew a part too big, but in the PS it can be directly select it and make it smaller." Similarly, Participant 45 comments: "Being able to quickly 'control z', or rub out an aspect of a drawing made the sketching process easier and faster than if using pen and paper. Additionally, being able to move aspects of a drawing around was useful when exploring ideas."

With respect to $\mathrm{CAD}$, there are a higher frequency of responses that cite software as an inhibiter (negative), for example, Participant 4 comments: "It was not easy to modify my design using Rhino because I was still not proficient to use new skills." With respect to sketching, the number of instances of easy modification is consistent with expectations on how sketching should be used, i.e. early in the design process where levels of detail tend to be low. Comments such as Participant 5 "It was not so hard to modify and change my rough sketches", And Participant 8, "not easy but is less complicated than other tools" are indicative of sketching being used early in the project.

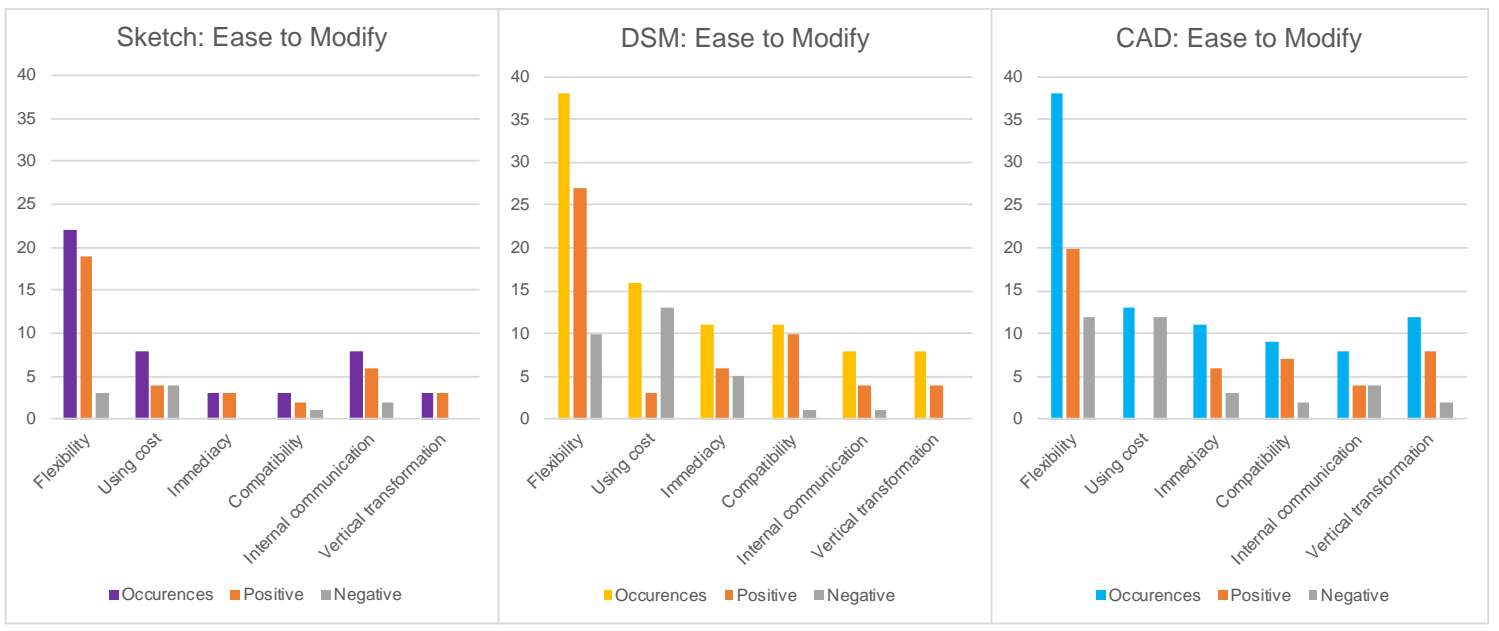

Figure 5. Comparison of most frequently cited DTCs along with respective proportion of positive versus negative attitudes describing each DTC on the topic of easy modification 
A similar pattern in diary entries describing Using cost to those discussing ease of visualisation (4.3) is also seen when discussing modification. Namely that Using cost tends to be more significant as the level/capacity of software increases (in DSM and especially in CAD). Closely linked to Flexibility and modification is the DTC Vertical Transformation. Most mentions in CAD, less in sketch and DSM. This is unsurprising as CAD is the primary tool for this type of detailing. What is notable is citations with respect to CAD have some negative attitudes whereas sketch and DSM are entirely positive. This suggests that the sketch-based input and resulting flexibility are helpful when making modifications when detailing designs. Lower frequency of mentions would suggest this is only the case up to a point. I.e. there is only so much detailing that is done with these tools but when it is done it facilitates relatively easy modification.

\subsection{Implications on combination of complementary affordances}

Fidelity is a key concern when visualising ideas. With respect to DSM combining affordances, results show the way that including crude 3D CAD representations (the proxy model) is helpful in facilitating easy visualisation in providing a guideline, but without the time investment (Using Cost). Thus, we have established support for the proxy model as a mechanism to deliver an increased level of fidelity (viewed positively by participants) illustrating its utility in easy visualisation.

Citations of Flexibility with respect to ease of visualisation highlight the way that DSM shows a promising inclusion of digital tools. We can see that the ability to undo, and manipulate designs is helpful in terms of modification, similar to the way that CAD offers such flexibility (J. A. Self 2013). However, we see fewer comments on limitations based around software complexity and fewer negative attitudes with respect to Using cost. This focus on Flexibility aligns closely with diary entries discussing ease of modification. Here, citations of Flexibility become more negative as designers progress through their design process using sketch, DSM and CAD. This is likely related to the stage of design that these tools are typically used, and the relative level of detail being designed. When using $\mathrm{CAD}$, designs are embodied in a greater level of detail which likely influences the ease of changing designs. The increasing number of citations of Vertical Transformation as participants progressed through Sketch, DSM and eventually CAD supports this explanation. Nevertheless, this trend shows that DSM has a level of easy modifications somewhere between that of Sketching and CAD.

In terms of combining complementary affordances, We can see that key characteristics of Fidelity and Flexibility show how DSM as a hybrid design tool offers substantially easier visualisation through the use of the proxy model. The inclusion of software is clearly helpful for modification of designs, however the effectiveness of software is likely to be heavily influenced by the stage in the design process and associated level of detail included in the design. Figure 6 illustrates the use of DSM in terms of the patterns of use and affordances shown in Figure 1. Hence, we contend the blend of sketch-like flexibility within digital sketching environment is helpful in providing easy visualisation of ideas at higher fidelity than sketching but with less of the software-based difficulties of CAD.
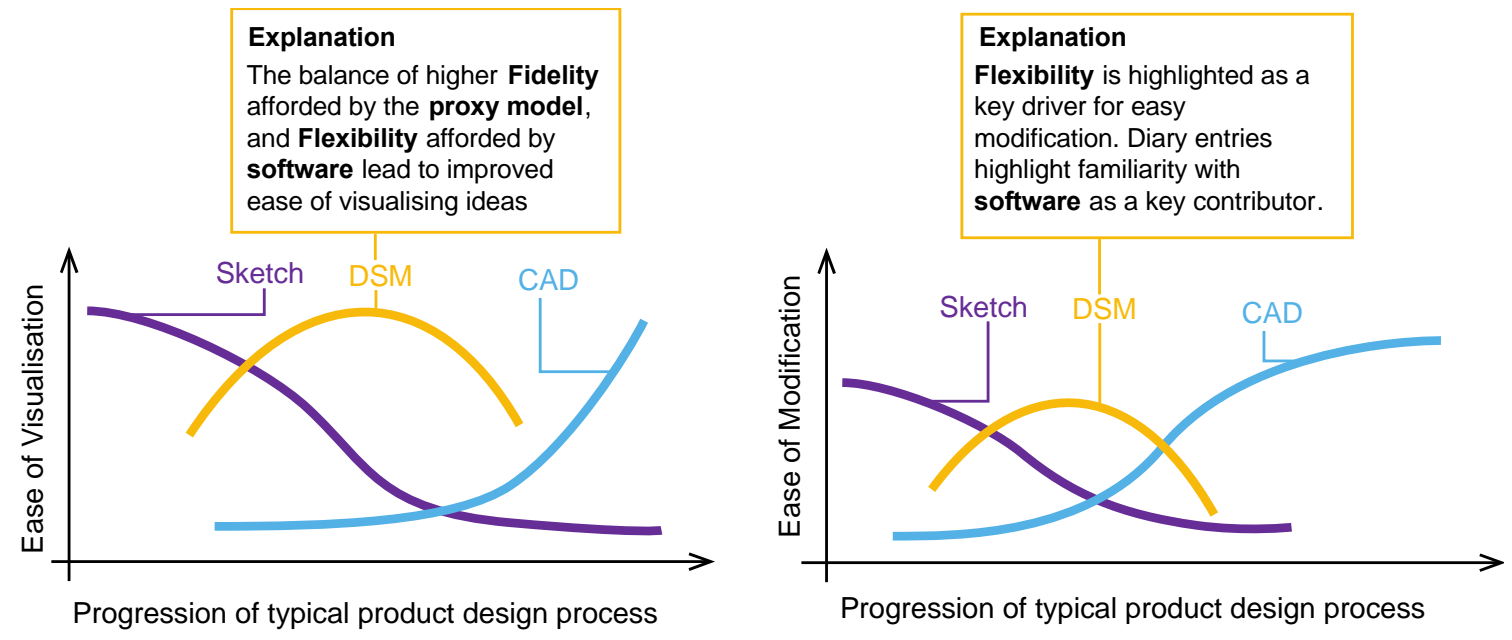

Figure 6. Illustrating key findings on the use of DSM in the context of typical design process progression 


\section{LIMITATIONS AND FURTHER WORK}

The primary limitation in this study relates to the use of diary entries to record data. Selection of the diary entry method was made so as to facilitate a large sample of participants (62) and keep intrusion on natural design process and the experimental bias that this can cause to a minimum. The compromise in using this method is limitation on the granularity of precise moments/steps in the design process to which diary entries refer. Although, participants followed a common process in terms of the sequence of tools used during broad phases (i.e. sketching in ideation, DSM in conceptualisation and CAD in design refinement), it is not possible to use this dataset to pin-point key design steps or visualisations for which comments in diary entries refer. Described in the context of Figure 1 and Figure 6, while we can validate that there is evidence supporting the use of DSM between sketching and CAD, we can't yet go further in specifying exactly when in the progression of typical design process, the optimum use occurs.

Thus, having established on a broad sequential level when the complementary affordances of DSM are beneficial to industrial and product designers, further work is required to understand in more detail precisely when DSM is most beneficial. We anticipate this would be done following methods used by (Alcaide-Marzal et al., 2013) where a smaller sample of participants observed and surveyed directly after shorter more specific design tasks. Such a study would also provide an opportunity to test the use of DSM in different design tasks varying level of technical or mechanical design involved, and level of constraint in briefs as these would no doubt influence attitudes on the utility of DSM. Furthermore, such a study could also provide a basis to assess the quality of resulting design.

\section{CONCLUSION}

This paper presents a study investigating the extent to which Digital Sketch Modelling (DSM) as a hybrid visualisation tool can deliver the complementary affordances of sketching and CAD, namely both easy visualisation as well as easy modification. A qualitative study of diary entries made by 62 postgraduate designers over the course of a design project formed the basis of the study. Analysis of the diary entries illustrated how the use of proxy models and the capacity of editing software in DSM resulted in substantially positive attitudes on its affordance for easy visualisation of ideas. Findings with respect to the affordance for easy modification of designs show the blend of software used led to fewer concerns about the level of time involved in making modifications than when using CAD software. Notably however diary entries showed how ease of modification is closely tied to stage in the design process. As such, while it is possible to conclude that DSM is beneficial as a transition tool between sketch and CAD for modifying designs, further work is required to define the precise type of design modification where DSM is more beneficial to the user than sketching or CAD.

In terms of the study's aim, we can conclude that DSM does deliver both complementary affordances of sketching and CAD, yet the positive influence of doing so is skewed more in the visualisation of ideas than the modification of ideas. Thus, we recommend the use of DSM earlier in the design process and even in very early CAD phases. However, the utility of DSM falls off as designs become more detailed at which point CAD is the ideal tool to use.

More broadly the study illustrates that the use of software in visualisation can offer benefits in flexibility, but that increased complexity of software can significantly hinder ease of modification and visualisation. Many negative reflections on CAD cited the way that software familiarity can inhibit both visualisation and modification of designs. Hence while inclusion of software alongside sketching (as used in DSM) has benefits, it is important to limit or curate the level of detail and complexity available to the user.

\section{REFERENCES}

Alcaide-Marzal, J., Diego-Más, J.A., Asensio-Cuesta, S. and Piqueras-Fiszman, B. (2013), “An exploratory study on the use of digital sculpting in conceptual product design", Design Studies, Vol. 34 No. 2, pp. 264 284, https://doi.org/10.1016/j.destud.2012.09.001.

Aldoy, N. and Evans, M. (2011), "A review of digital industrial and product design methods in UK higher education”, The Design Journal, Vol. 14 No. 3, pp. 343-368, available: https://doi.org/10.2752/175630611×13046972590923

Arora, R., Habib Kazi, R., Grossman, T., Fitzmaurice, G. and Singh, K. (2018), "SymbiosisSketch: Combining 2D \& 3D Sketching for Designing Detailed 3D Objects in Situ", in Proceedings of the 2018 CHI 
Conference on Human Factors in Computing Systems, ACM, Vol. 185, https://doi.org/10.1145/3173574.3173759

Badke-Schaub, P. and Frankenberger, E. (1999), “Analysis of design projects”, Design Studies, Vol. 20 No. 5 , pp. 465-480, https://doi.org/10.1016/s0142-694x(99)00017-4

Bae, S.-H., Kim, W.-S. and Kwon, E.-S. (2003), "Digital Styling for Designers: Sketch Emulation in Computer Environment" in, 987-987.

Company, P., Contero, M., Varley, P., Aleixos, N. and Naya, F. (2009), “Computer-aided sketching as a tool to promote innovation in the new product development process", Computers in Industry, Vol. 60 No. 8, pp. 592-603, https://doi.org/10.1016/j.compind.2009.05.018

Cross, N. (1982), “Designerly ways of knowing”, Design Studies, Vol. 3 No. 4, pp. 221-227, https://doi.org/10.1016/0142-694x(82)90040-0

Evans, M.A., Pei, E., Cheshire, D. and Graham, I.J. (2015), "Digital sketching and haptic sketch modelling during product design and development", https://doi.org/10.1504/ijpd.2015.069323.

Goldschmidt, G. (1991), "The dialectics of sketching", Creativity research journal, Vol. 4 No. 2, pp. 123-143, https://doi.org/10.1080/10400419109534381.

Kara, L.B., D'Eramo, C.M. and Shimada, K. (2006), "Pen-based styling design of 3D geometry using concept sketches and template models", in Proceedings of the 2006 ACM symposium on Solid and physical modeling, Cardiff, Wales, pp. 149-160, http://doi.acm.org/10.1145/1128888.1128909.

Kara, L.B. and Shimada, K. (2008), "Supporting early styling design of automobiles using sketch-based 3D shape construction”, Computer-Aided Design \& Applications, Vol. 5 No. 6, p. 9, https://doi.org/10.3722/cadaps.2008.867-876.

Lawson, B. (2002), “CAD and creativity: does the computer really help?”, Leonardo, Vol. 35 No. 3, pp. 327 331 https://doi.org/10.1162/002409402760105361.

Orbay, G. and Kara, L.B. (2012), "Shape Design From Exemplar Sketches Using Graph-Based Sketch Analysis", Journal of Mechanical Design, Vol. 134 No. 11, p. 111002, https://doi.org/10.1115/1.4007147

Pedgley, O. (2007), "Capturing and analysing own design activity”, Design Studies, Vol. 28 No. 5, pp. 463-483, https://doi.org/10.1016/j.destud.2007.02.004.

Purcell, A. and Gero, J.S. (1998), "Drawings and the design process: A review of protocol studies in design and other disciplines and related research in cognitive psychology", Design Studies, Vol. 19 No. 4, pp. 389430, https://doi.org/10.1016/s0142-694x(98)00015-5.

Ranscombe, C. and Bissett-Johnson, K. (2017), "Digital Sketch Modelling: Integrating digital sketching as a transition between sketching and CAD in Industrial Design Education", Design \& Technology Education, Vol. 22 No. 1.

Self, J., Dalke, H. and Evans, M. (2009), “Industrial Design Tools and Design Practice: An approach for understanding relationships between design tools and practice".

Self, J.A. (2013), "CAD Tools and Creative Design, Grounds for Divorce or Match Made in Heaven?" CAD/CAM Review, Vol. 19 No. 2, pp. 36-43.

Tsai, G. and Yang, M.C. (2017), "How It Is Made Matters: Distinguishing Traits of Designs Created by Sketches, Prototypes, and CAD”, in ASME 2017 International Design Engineering Technical Conferences and Computers and Information in Engineering Conference, American Society of Mechanical Engineers, available: https://doi.org/10.1115/detc2017-68403.

Zammit, R. and Munoz, J.A.I. (2014), "Has Digital Clay Finally Arrived?", Computer-Aided Design and Applications, Vol. 11 No. sup1, S20-S26, https://doi.org/10.1080/16864360.2014.914402.

Zhang, W., Ranscombe, C., Radcliffe, D. and Jackson, S. (2019), "Creation of a framework of design tool characteristics to support evaluation and selection of visualisation tools". Paper presented at the 22nd International Conference on Engineering Design (ICED), Delft, Netherlands, Article in press. 